\title{
Defects detection on TFT lines of flat panels using a feed forward neural network
}

\author{
H.A. Abeysundara ${ }^{1}$, Hiroshi Hamori ${ }^{1}$, Takeshi Matsui ${ }^{2}$, Masatoshi Sakawa ${ }^{2}$ \\ 1. Research and Development Division, OHT Incorporation, Fukuyama, Japan. 2. Department of Electrical Systems and \\ Mathematical Engineering, Faculty of Engineering, Hiroshima University, Japan.
}

Correspondence: H. A. Abeysundara. Address: Research and Development Division, OHT Incorporation, $1118-1$ Nishichujo, Kannabe-Cho, Fukuyama 720-2123, Japan. Email: ha_abey@ohtinc.jp.

Received: January 15, 2013

Accepted: March 11, $2013 \quad$ Online Published: May 24, 2013

DOI : $10.5430 /$ air.v2n4p1

URL: http://dx.doi.org/10.5430/air.v2n4p1

\begin{abstract}
This paper proposes a novel approach for inspection of open/short defects on thin film transistor (TFT) lines of flat panel displays (FPD). The inspection is performed on digitized waveform data of voltage signals that are captured by a capacitor based non-contact sensor by scanning over TFT lines on the surface of mother glass of FPD. The sudden deep falls (open circuits) or sharp rises (short circuits) on the captured noisy waveform are classified and detected by employing a four-layer feed forward neural network with two hidden layers. The topology of the network consist of an input layer with two units, two hidden layers with two and three units respectively and an output layer with one unit and a standard sigmoid function as the activation function of each unit. The network is trained with a fast adaptive back-propagation algorithm to find an optimal set of associated weights of neurons by feeding a known set of input data. This method is an alternative to the existing thresholding based non-contact method which has always its own limitations and drawbacks due to some non-avoidable features of input data such as non-stationary patterns and varying magnitude levels at defect points. Experimental results show that this method can adapt fast for new input patterns and avoids the ambiguity of threshold definitions and therefore it is more feasible than the existing thresholding method.
\end{abstract}

\section{Key words}

Feed forward neural networks, Error back-propagation, Flat panel display, Open short detection, Non-contact defect inspection

\section{I ntroduction}

With the remarkable growth of demand for flat panel displays in recent years, having a wide verity of applications such as televisions, computers, cameras, hand phones, medical equipments, toys and etc, there is a stiff competition among manufacturers for high throughput product lines and low priced manufacturing. The demand for larger sizes of mother glass (e. g. Generation 10, $11 \& 12$ ) and high density thin film transistor (TFT) pitch patterning of FPDs have also been increasing. However when the pitch pattern density of panels is increasing the tendency of defects such as inter layer short circuits between TFT lines is also increasing. Therefore recently, manufactures have been forced to keep significant importance on detection of defects in early stages of production process of FPDs and repair them early. As a result, the speed and the precision of defects detection have been major issues for the manufactures of FPDs and researchers as well. 
Automatic optical inspection (AOI) methods, which are based on still or video images, have been mainly used in the past for detection of defects during intermediate processes of fabrication lines of FPDs ${ }^{[5,6]}$. Though the AOI methods, being based on images, are fast, non-contact and no damage occurs to glass substrates like pin probe methods, non-electrical defects such as particles on panel surface (micro dusts) and even slight color changes on TFT wirings can also be falsely detected as defects. A major drawback in AOI methods is that it is extremely difficult to distinguish all those non-electrical defects from electrical defects (open NG and short NG) that need to be repaired and restored. Furthermore, some common electrical defects, which occur with the increased pitch patterning, such as cracks on wirings and short circuits in between layers under wire crossings cannot be detected properly with AOI methods. In general, workers operating such repair systems are required to visually check all such defects detected by the inspection system and to judge whether a repair is needed or not which is tedious and cumbersome. Therefore to avoid operators being involved in such unnecessary work, it is necessary to improve the efficiency and accuracy of detecting electrical defects.

However, if it is only for detecting electrical defects then there is the commonly used pin probed inspection method of which electrode pins make direct contacts on each and every wiring on the panel surface and measure the current flown after applying some voltage. Though this method has the advantage of detecting nothing but electrical defects, it also has disadvantages such as very low-speed of inspection, poor adjustability for the changes of TFT circuit design and line pitch and the necessity of frequent replacing of pin probing fixtures of the inspection machines which is an expensive process.

Non-contact FPD inspection method proposed by Hamori et al. ${ }^{[1-4]}$ is the most promising technique so far, which is totally non-contact, utilizing a capacitor based sensor that scans over the TFT lines of mother glass panels of FPDs. The detection of defects in that system becomes detection of sharp rises or deep falls on a waveform of a voltage signal captured by the sensor. The method they use to detect those defective points on waveforms is a thresholding method after some noise cleaning operations. However determining a proper threshold to correctly indentify those rises or falls on waveforms is still not easy since such measured voltage signals contain various noises such as random noises, external vibrations and other noises due to environmental effects such as machine temperature.

Hence by looking at literature there are three main methods of defects inspection on flat panels, firstly the AOI method which is based on image processing using techniques such as wavelet transformation or singular value decomposition ${ }^{[5,6]}$. Secondly the direct pin probe method which is slow and expensive and involves no data processing and finally the capacitor based non-contact inspection method that employs a thresholding technique to search defects on voltage waveforms. All of those are techniques based on some kind of thresholds and there is no learning or experience involved.

Therefore, in this paper, we propose an alternative detection method using an artificial neural network, which is novel to the area of defects detection on flat panels, to the above mentioned non-contact method proposed by Hamori et al ${ }^{[1,2]}$. Since the problem is highly data driven and non-linear on a set of non-stationary waveform data, an intelligent approach must be more appropriate which can learn and adapt according to varying patterns of data. We have adopted a 4 layered feed forward neural network to classify candidate points, which are selected from waveform data and presented to the network, as defective (NG) or non-defective (OK). There is no threshold selection ambiguity involved in this method instead some local and neighborhood characteristics of candidate points are entered as inputs to the network and the network itself determines if it is defective or not based on prior learning. There are three local features currently used as such inputs which are Signal to noise ratio (SNR), Residual difference and Change of wave length at a given particular candidate point and its neighborhood. The network is trained using an adaptive error back-propagation algorithm ${ }^{[7]}$ based on gradient descent by feeding a known set of hand picked input data comprising every possible pattern of defective and non-defective points on the waveform. Experimental results show that this method is much more feasible than the existing thresholding method. 


\section{Non-contact inspection}

As illustrated in Figure 1, in the non-contact defects inspection method proposed by Hamori et al. ${ }^{[1-4]}$, the capacitor based non-contact sensor utilizes two electrodes, a feeding electrode and a receiving electrode, that scan parallel to each other across TFT lines over the mother glass of FPD panel. During scanning, a small voltage is applied into TFT lines on the panel through the feeding electrode and is received through the receiving electrode that captures the voltage signal through an $\mathrm{AD}$ converter to the host computer as a digitized waveform and taken into analysis.

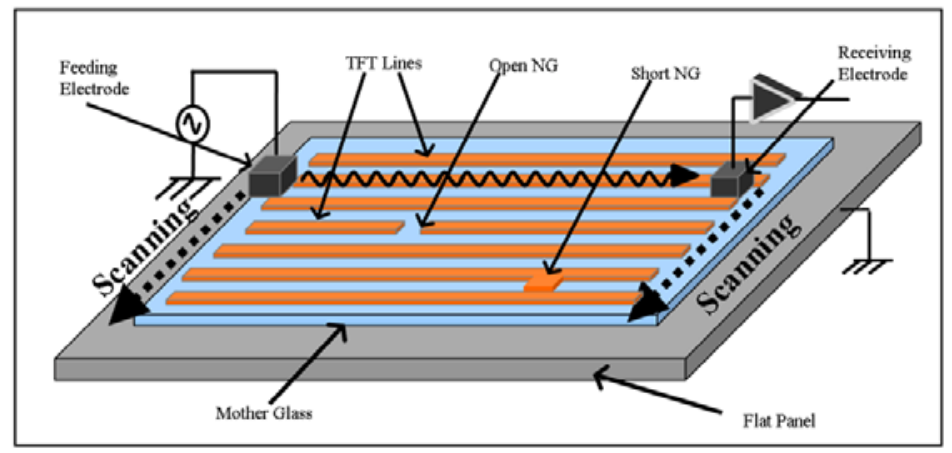

Figure 1. The Non-contact FPD inspection system

Figure 2 shows such a typical waveform pattern of a captured voltage waveform through a non-contact sensor. The electrical defects on TFT lines (open NG or short NG) will show sudden sharp rises or deep falls on the waveform and therefore detection of defects would become detecting those rises and falls on the waveform. Generally such waveforms are mixed with lot of random noises, external vibrations and other artifacts as shown in the figure. The large deviation at point $a$ may be a random electrical noise, at point $b$ may be a deviation caused due to a real electrical defects and at point $c$ may be a vibration caused by an external force. Due to practical reasons in real production environments the gap between the surfaces of the scanning electrodes and the flat panel are not uniformly even. This unevenness causes low frequency swinging or baseline fluctuations on the captured voltage waveform as visible in the figure.

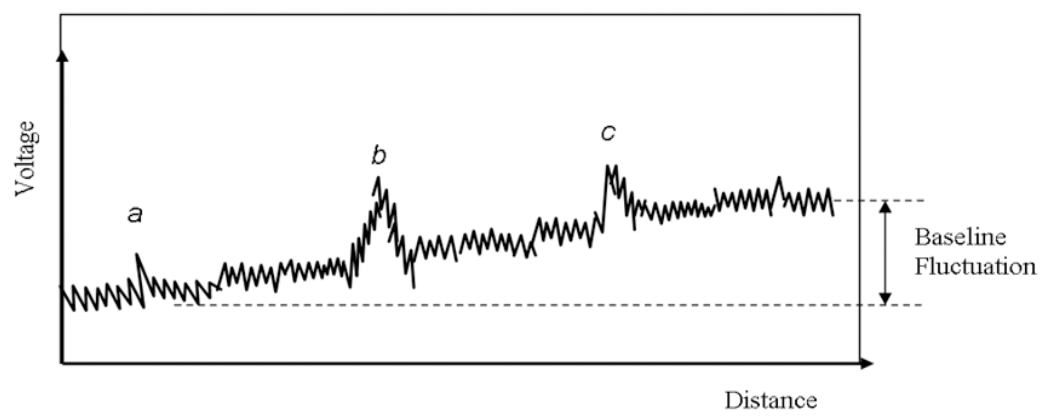

Figure 2. A typical pattern of a waveform captured by a non-contact sensor

\subsection{Detection of defects by thresholding - existing method}

As described above, since the original waveform data captured by non-contact sensors are full of noises (see Figure 3a), a moving average filter is applied initially to reduce high frequency random noises (see Figure 3b). For example if a waveform $f$ of sample size $N$ is given, the resulting waveform $g$ after a moving average filter with a pre-determined subset size $p$ is in the form of: 


$$
g_{n}=\frac{f_{n}+f_{n+1}+\ldots .+f_{n+(p-1)}}{p}: n=1, \ldots, N-p
$$

where $f_{n}$ is the waveform value of $f$ at point $n$ and $g_{n}$ is the waveform value of $g$ at point $n$. Then the low frequency swinging and baseline fluctuations of the waveform due to the unevenness of the gap between the panel surface and the sensor surface are neutralized by applying a derivative operator (Figure 3c). If the waveform after a derivative operator of step length $s$ is $h$, then $h$ can be expressed as:

$$
h_{n}=g_{n+s}-g_{n} ; n=1, \ldots ., N-s .
$$

The resulting waveform is undergone again a moving average operator to remove remaining spike noises. Finally magnitude values of the waveform are compared with a pre-determined threshold value and the points that exceed the threshold level are considered as defect points (Figure 3c).

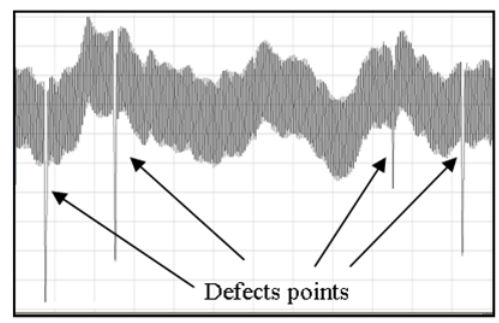

(a)

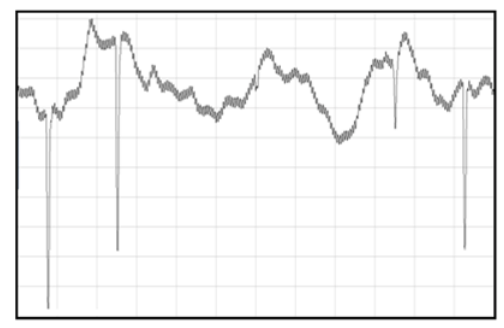

(b)

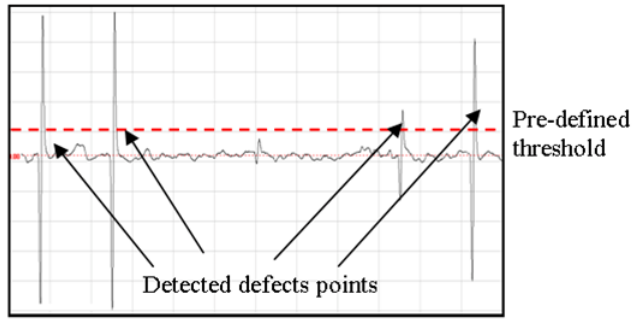

(c)

Figure 3. Detection of defects by thresholding method: (a) Original waveform, (b) After noise suppression, (c) Thresholding on differential waveform

\subsection{Drawbacks in the thresholding method}

The thresholding method described in 2.1 is quiet appropriate as long as environmental effects such as the machine temperature and factory vibrations and the gap between surfaces of the sensor and the panel remain firmly stationary, which minimizes extra noises and unnecessary deviations on the voltage waveform. However in real production situations in factories the temperature and the external vibrations can vary from time to time and from machine to machine. It can also be varied from location to location of machines even inside the same factory. Also keeping the distance between the sensor and the FPD panel fixed during scanning is not an easy task so that the voltage signal shows fluctuations or swinging with the change of the gap. All of these factors severely affect the pattern and the noise level of the captured voltage waveform and determining threshold parameters for the software program is a difficult task as shown in Figure 4. Whenever an inspection machine is changed or its location is changed, operators have to look carefully several waveforms and set threshold parameters manually. Furthermore even in a fixed environment and on a fixed single machine, patterns and noise levels can still be varied from channel to channel (there are 4 or 8 channels that perform scanning simultaneously over the FPD panel).

On the other hand, the deciding factor used for determining defective points on a waveform, i.e. the threshold parameter set by the operator, is a global value which applies to the entire waveform. However most of the above effects to the voltage signal are local effects on respective waveforms, in which, taking a global threshold value as the deciding factor in a highly locally dependent feature space is lacking appropriateness.

As shown in Figure 4, the points B, C and E in the left side graph are real defect points and the points A and D are not defects but sudden fluctuations due to change of gaps between the sensor and the panel. The corresponding points for real 
defects on the differentiated waveform (right side graph) are B', C' and E'. Out of these 3 defect points only B' and E' can be detected using the threshold and point $\mathrm{C}^{\prime}$ is difficult to be detected by the threshold although it is actually a defect point. If the threshold level is further lowered the miss-detected point C' can be detected but in the same time points A' and D' can also be detected as defects, which will be false detections. In manufacturers stand point both miss-detections and false detections are costly.

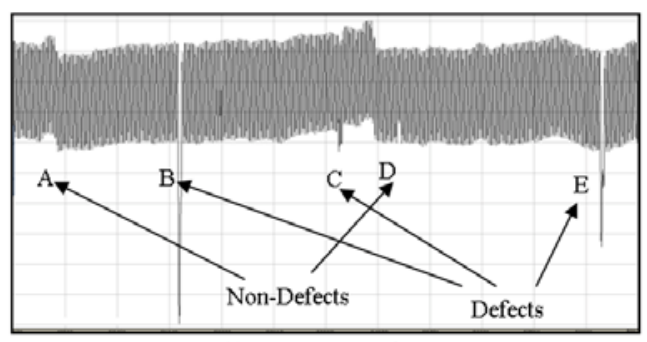

(a) Original waveform

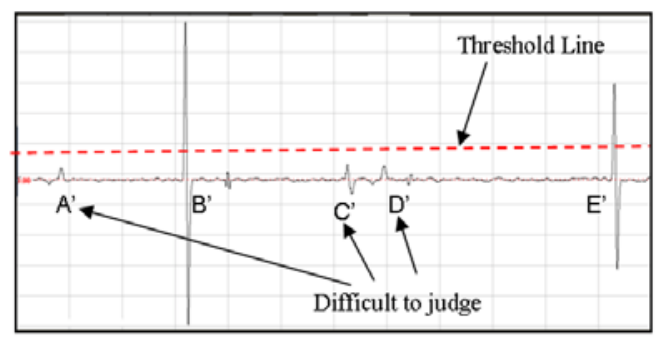

(b) Differentiated waveform

Figure 4. Difficulty of determining a proper threshold value

This hurdle of determining a proper threshold value is difficult to leap over as long as the threshold value is global and the features around real defect points are local. The lower the threshold value is set the higher the ratio of false detections appear whereas the higher the threshold value is set the higher the ratio of miss-detections occur. This is a major barrier in the existing thresholding method to achieve a superior level of performance avoiding both miss-detections and false detections.

\section{Proposed method using a feed forward neural network}

\subsection{Why a neural network}

As described in the section 2.2, the existing criteria of using a threshold for determining defect points on waveforms in non-contact defect inspection method proposed by Hamori et al. is lacking the appropriateness since the feature variations on and around defect points are largely local features while the deciding threshold is a global value. For identifying such variations on such a complex space, it requires a fast adaptive algorithm with a high degree of accuracy and efficiency since the system is largely data driven and the patterns of defect points on waveforms are highly non-linear. The cross correlation method can be used to detect such patterns on waveform data which is a measure of 'goodness of fit' with a pre-selected pattern. The level of fitness of $80 \%$ or more corresponds to patterns in data that are easily discerned as good matches by the human eye. However having various patterns of waveforms with various patterns of defect points on them it requires to prepare and store hundreds of patterns if not thousands, which would be a huge time consuming exercise. Moreover the features around defect points on waveforms are varied from channel to channel, from location to location and etc. So that a technique such as a neural network that can learn the environmental effects and memorize, must be a much suitable way of addressing the problem. An artificial neural network is an intelligent agent that can handle effectively a large amount of dynamic, non-linear and noisy data ${ }^{[7-9]}$. It also can observe, learn and memorize from the experience before performing a particular task. Therefore the most reliable approach must be such an intelligent approach such as using a neural network since a neural network can be trained by feeding known data before actually put into perform and can keep the adaptability. Under these contexts we have tested numerous topologies of neural networks and adopted a 4-layer feed forward neural network as described in next sections.

\subsection{I nputs to the feed forward neural network}

Since a neural network can learn from experience, i.e. a neural network can be trained by feeding a known set of data, any feature around an input point on the input waveform that can be considered as influential to the output must be considered 
as an input to the network. By looking at neighborhood characteristics around defective points on waveforms and comparing with normal area, following three features have been identified as inputs to the network (input vector $\underline{x}$ ), namely Signal to Noise Ratio (SNR), Residual difference, and Change of wave length. All of these input parameters $x\left(x_{1}, x_{2}, x_{3}\right)$ are picked within a pre-determined length of neighborhoods of possible candidate points on the waveform whereas candidate points are selected by a simple low level threshold such that it may include many false detection but not miss-detections

\section{Signal to noise ratio}

If the characteristics within a neighborhood around a defect point on the waveform are observed (see Figure 5) it is understood that there is a sharp deviation of magnitude from the normal area. In other words the level of the signal at a particular point shows a considerable deviation against the level of background noise, which means a change of signal to noise ratio (SNR). Therefore SNR is considered as the first component $\left(x_{1}\right)$ of the input vector $\underline{x}$ and is taken as:

$$
x_{1}=S N R=\frac{\mu}{\sigma}
$$

where $\mu$ is the mean value and $\sigma$ is the standard deviation of the waveform within the selected neighborhood.

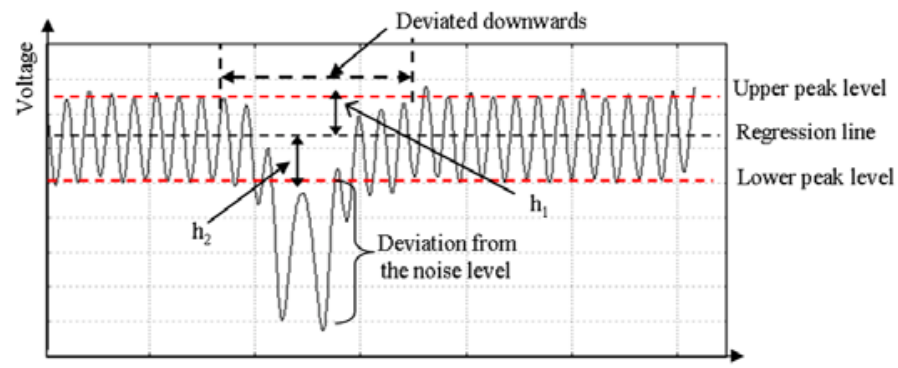

Figure 5. Measurement of SNR and Residual Difference in a neighborhood of a defect point

\section{Residual difference}

Besides the sharp deviation at the defect point, the neighboring area consisting of a few wave lengths can also be seen deviated towards the same direction as main deviated point (see Figure 5). This particular feature of the waveform within the neighborhood is measured as the difference of average upper peak level with the regression line $\left(h_{1}\right)$ and the difference of average lower peak level with the regression line $\left(h_{2}\right)$. In other words the residual difference of upper and lower peek levels in the neighborhood is taken as the second component $\left(x_{2}\right)$ of the input vector $\mathrm{x}$ and is taken as:

$$
x_{2}=h_{1}-h_{2}
$$

\section{Change of wave length}

In the original waveform it shows a considerable change of wave length at a defect point (see Figure 5) and is taken as the next input to the network. Though this wave length change appears in the normal waveform, it is easier to measure on the differentiated waveform as seen in Figure 6. So that the rate of change of wave length of a defect point from that of average wave length in the normal area is taken as the third component $\left(x_{3}\right)$ of the input vector $\underline{x}$ and is taken as:

$$
x_{3}=\frac{D-d}{d}
$$

where $D$ is the wave length at the input point and the $d$ is the average wave length at neighborhood (see Figure 6). 


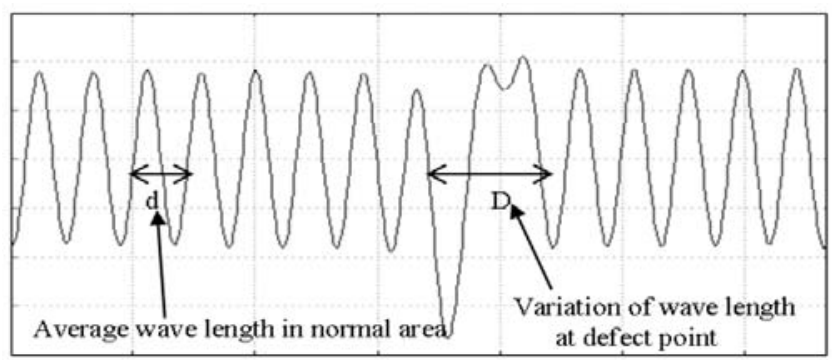

Figure 6. Neighborhood of a defect point in differentiated waveform

\subsection{Topology of the feed forward neural network}

Determining the best topology of neural network for a certain problem is also an important task and hence we tested various topologies with both single hidden layer and two hidden layers. Convergence pattern and convergence limit of the error graph during error backpropagation of many of them were not as expected as described and shown in section 4.1. Most of their error convergence levels were much higher or the error curve fell rapidly but converged to a higher level. However After testing numerous topologies of neural networks we have found that the most reliable network for our problem is a 4-layer feed forward network such that an input layer with two units, two hidden layers with 2 units and 3 units respectively and an output layer with one unit (see Figure 7). The input feature vector $\underline{x}\left(x_{1}, x_{2}, x_{3}\right)$ consists of 3 components (above mentioned 3 input parameters), the weight space consists of 27 weights which is inclusive of weights associated with bias input to each unit which are not depicted in the figure and the output vector y is single component. The total network function Net can then be represented as:

$$
\underline{y}=\operatorname{Net}(\underline{x})
$$

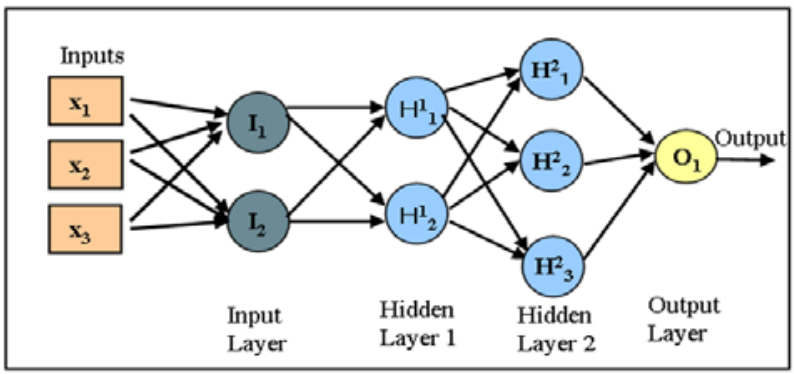

Figure 7. Topology of the feed forward neural network

\subsection{Activation function}

The activation function of each computing unit in the network is in the form of a sigmoid function (logistic function) because training of the network is to be done by error back-propagation algorithm which requires the activation function to be a continuous function.

Since the back-propagation requires computation of gradients of the error function at each iteration step, the continuity and differentiability of the error function must be guaranteed. Therefore the activation function $f$ of each unit in the network is taken as:

$$
f(X)=\frac{1}{1+e^{-X}} \text { and } \quad X=\sum_{i=1}^{n} w_{i} X_{i}
$$


where $x_{i}(i=1, . ., n)$ are the inputs to the unit (in case of input layer those are the components of the input vector $\underline{x}$, and in case of a hidden or output layer those are outputs from the previous layer) and $w_{i}$ are the weights associated with each such input.

Then the calculation of an output value $y$ from the above network can be explicitly expressed as:

$$
y=f\left(\sum_{l=1}^{3} w_{o l} \cdot\left(f\left(\sum_{k=1}^{2} w_{l k} \cdot\left(f\left(\sum_{j=1}^{2} w_{k j} \cdot\left(f\left(\sum_{i=1}^{3} w_{j i} \cdot x_{i}+B_{j}^{I}\right)\right)+B_{k}^{H 1}\right)\right)+B_{l}^{H 2}\right)\right)+B^{O}\right)
$$

where $w_{j i}$ is a weight in input layer connecting $i^{\text {th }}$ input and $j^{\text {th }}$ neuron, $w_{k j}$ is a weight in hidden layer 1 connecting $j^{\text {th }}$ neuron in the input layer and $k^{\text {th }}$ neuron in the hidden layer $1, w_{l k}$ is a weight in hidden layer 2 connecting $k^{\text {th }}$ neuron in the hidden layer 1 and $l^{\text {th }}$ neuron in the hidden layer 2 and $w_{o l}$ is a weight in the output layer connecting $l^{\text {th }}$ neuron in the hidden layer 2 and the output neuron. $B_{j}^{I}$ is the bias for the $j^{\text {th }}$ neuron in the input layer, $B_{k}^{H 1}$ is the bias for the $k^{\text {th }}$ neuron in the hidden layer $1, B_{l}^{H 2}$ is the bias for the $l^{\text {th }}$ neuron in the hidden layer 2 and $B^{O}$ is the bias for the output neuron. $X_{i}$ is the $i^{\text {th }}$ component of the input vector $\underline{X}$ and $f$ is the common activation function (5) of the network..

\subsection{Training the network}

A feed forward neural network is a computational graph whose nodes are computing units and whose directed edges transmit numerical information from node to node. In the network, each arrow from left side to right side (see Figure 7) is associated with a synaptic weight and those weight values must be optimized before use in a real situation task. The combination of those weights which minimizes the error is considered to be the optimal solution to the learning problem. Since there are many weights in the network associating each input in a layer to the each unit in the next layer, we don't know how much each of those weights is to blame for the final error and divvy up the adjustment among these weights proportionately. Therefore this problem can be called a blame assignment problem or a credit assignment problem. The back-propagation solves this problem, as its name depicts it looks for the minimum of the error function in weight space using the method of gradient descent. A set of known inputs comprising both NG points and OK points in all sorts of patterns of input data will be used as the training data set.

In general this process of error convergence takes long time for offline training when a large set of training data is used. Therefore we also have adopted an adaptation technique ${ }^{[7]}$ in order to accelerate the convergence of the error function. It is an adaptive step algorithm that the step size is increased whenever the algorithm proceeds down the error function over several iterations. When the algorithm moves over a valley of the error function the step size is decreased.

\section{Experimental results}

\subsection{Training results}

Training of the network was carried out with the error back-propagation algorithm by using a hand picked data set containing 50 defect points (NG) and 50 non-defect points (OK) covering every possible pattern of defect points and non-defect points as shown in Figure 8. The data were captured by OHT's three different GX-3 High speed LCD/PDP testers in different locations. The 3 input parameters (SNR, Residual difference and the Change of wave length) were picked from those selected data points and used as the input data set for offline training of the neural network. 


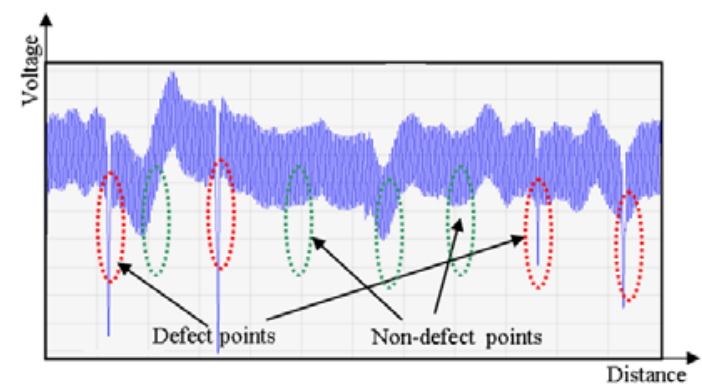

(a) Segment 1

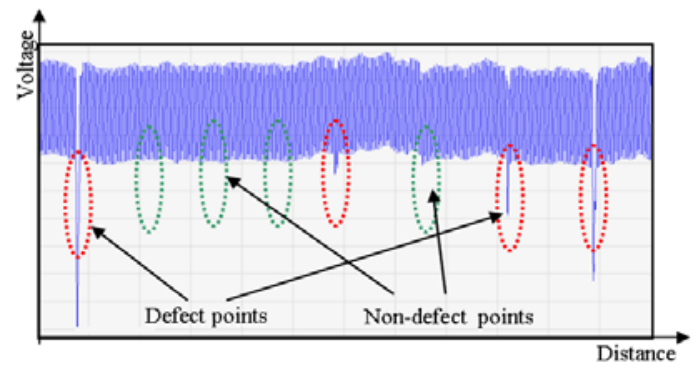

(b) Segment 2

Figure 8. Two segments of different waveforms used to pick data for network training

As described in section 3.3, numerous topologies of feed forward neural networks with both one hidden layer and two hidden layers were tested to determine the best one for our problem. Error graphs of most of them were either moved a little bit horizontally or fell suddenly and then in both cases converged to a higher level than the excepted level. Figure 9(a) and Figure 9(b) are two of such unacceptable error graphs with a single hidden layer consisting 3 units and 2 units respectively. As shown, their convergence levels are much higher than the level in our selected network with 2 hidden layers as shown in Figure 10, which converges until the given limit of 0.01 . Therefore a 4 layer network with 2 hidden layers was judged as the best topology for our problem as its error graph converged until the given small convergence limit.

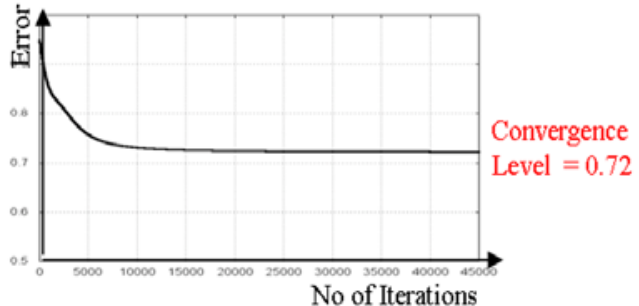

(a)

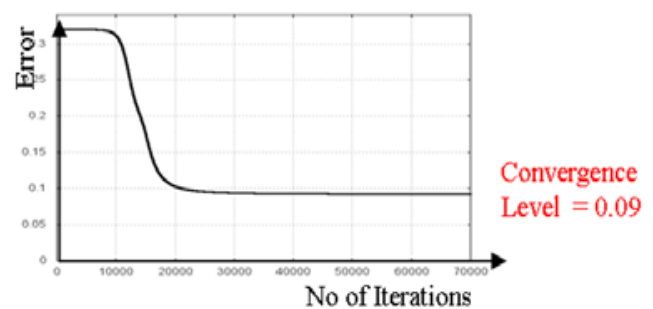

(b)

Figure 9. Error convergence in a single hidden layer network: (a) 3 neuron hidden layer, (b) 2 neuron hidden layer

Initially all the weight values of the above selected network were randomly set to small values between 0 and 0.1 and the learning constant $\gamma$ was set to 0.1 . The error back-propagation was performed iteratively until the error function converged to a level smaller than a pre-determined small value as shown in Figure 10. The convergence time of the error graph was considerably reduced by using an adaptive technique that adjusts the step length according to flow of the error function. Table 1 and Table 2 show the training results.

Table 1. Training parameters

\begin{tabular}{|l|l|}
\hline No of training data & 100 \\
\hline Initial weight values (Random) & $0 \sim 0.1$ \\
\hline Initial step size $(\gamma)$ & 0.1 \\
\hline Error convergence limit & 0.01 \\
\hline No of iterations & 54454 \\
\hline
\end{tabular}

As seen in Table 2, although the initial weight values were set between 0 and 0.1 the final optimized weight values, after error back-propagation, range from even smaller values to even bigger values. A positive weight represents an excitatory connection whereas a negative weight represents an inhibitory connection. 


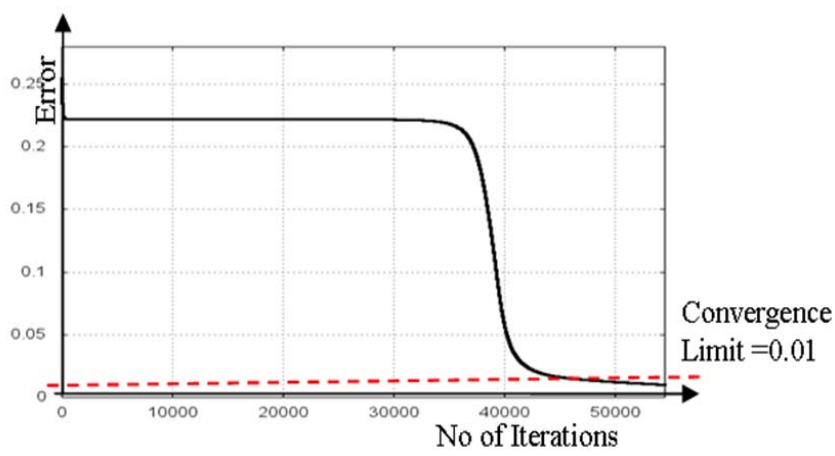

Figure 10. Convergence of error graph in back-propagation

Table 2. Optimized network weights after error back-propagation

\begin{tabular}{llll}
\hline Input Layer & Hidden layer 1 & Hidden layer 2 & Output layer \\
\hline 0.4299 & -0.0869 & 0.4477 & -0.5639 \\
-0.2330 & -1.5049 & 0.153 & -1.1827 \\
-0.1928 & 3.7513 & 3.7782 & 3.6198 \\
0.0507 & -3.1422 & -0.5832 & 3.7065 \\
1.7997 & 0.4497 & -1.6563 & \\
-0.5134 & -0.2282 & 3.8409 & \\
0.2399 & & -0.5956 & \\
0.1892 & & -1.6760 & \\
& & 2.2174 & \\
\hline
\end{tabular}

\subsection{Detection results}

The trained feed forward neural network (with parameters shown in Table 2) was used to detect defect points on waveform data captured by a non-contact sensor scanned over TFT lines of flat panels. A simple moving average filter was initially applied to smooth waveform data before picking candidate points to the network. Then candidate points were selected using a lower threshold value than the threshold value used in ${ }^{[1,2]}$ and the three input parameters to the network were picked from those candidate points and entered into the network. Figure 11 shows some detection results on 3 different waveforms captured from 3 different machines in different locations. All of those voltage waveforms consist of different levels of random noises, external vibrations and baseline fluctuations on them but were able to detect using our method correctly. The points marked at dashed lines in Figure 11(a) (both red and blue) are candidates that were selected as inputs to the network, and there were 3 defects (at red dashed lines) detected correctly out of 10 candidates. Similarly Figure 11(b) shows 3 defects detected correctly out of 8 candidates and Figure 11(c) shows 2 defects detected correctly out of 26 candidates.
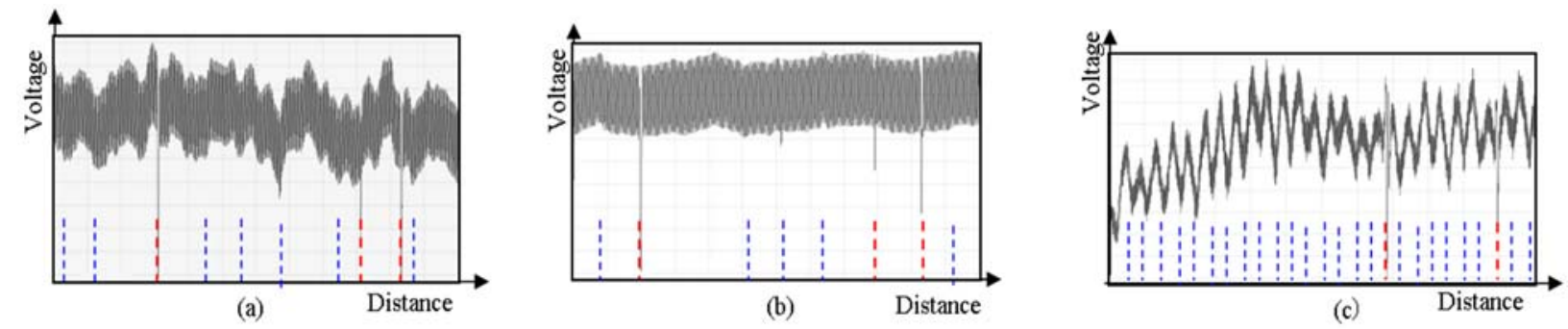

Figure 11. Detection results on 3 different waveforms captured by 3 different machines in 3 different locations 
Table 3 shows a comparison of miss-detections and false detections between our method and old method (existing thresholding method ${ }^{[1,2]}$ ). It shows comparison results for 3 different data sets captured from 3 different machines. The ratio of miss detections were able to reduce from existing $20 \% \sim 30 \%$ to around $5 \%$ and the ratio of false detections from existing $20 \% \sim 30 \%$ to below $15 \%$.

In addition, due to the reduction of ratio of false detections, the number of repairs was able slash drastically and due to the reduction of miss-detections, manual check ups were reduced and customer satisfaction was increased. Moreover, manual workload for threshold setting for operators, whenever the machine or the location or the product type to be tested is changed, was also decreased with the use of a trained network. As a result the overall testing time was improved and hence this method can be considered as fast and adaptive.

Table 3. Comparison of miss-detections and false detection between old and new methods

\begin{tabular}{|c|c|c|c|c|c|}
\hline \multirow{2}{*}{\multicolumn{2}{|c|}{ Total defects }} & \multicolumn{2}{|c|}{ Miss-detections } & \multicolumn{2}{|c|}{ False detections } \\
\hline & & Old method & New method & Old method & \multirow{2}{*}{$\begin{array}{l}\text { New method } \\
6(15.0 \%)\end{array}$} \\
\hline Data set 1 & 40 & $11(27.5 \%)$ & $3(7.5 \%)$ & $10(25.0 \%)$ & \\
\hline Data set 2 & 55 & $13(23.6 \%)$ & $3(5.4 \%)$ & $16(29.0 \%)$ & $7 \quad(12.7 \%)$ \\
\hline Data set 3 & 36 & $7 \quad(19.4 \%)$ & $1 \quad(2.7 \%)$ & $11 \quad(30.5 \%)$ & $5 \quad(13.8 \%)$ \\
\hline Total & 131 & $31 \quad(23.6 \%)$ & $7 \quad(5.3 \%)$ & $37 \quad(28.2 \%)$ & $18(13.7 \%)$ \\
\hline
\end{tabular}

\section{Conclusions}

In this paper, we proposed a novel defects detection method based on a 4-layered feed forward neural network to the problem of non-contact defect detection on flat panels in order to improve the short comings in the existing thresholding method. Our method does not use any global parameter (i.e. pre-determined threshold value) when determining the defect points on voltage waveforms captured by non-contact sensors. It only considers local features of the voltage waveform within a neighborhood of candidate points as input parameters to the neural network. Therefore the threshold selection problem does not arise and the function of the network would become almost similar to how the human eye observes the features of the waveform around defect points and determine if it is a defect or not.

To verify the performance of our method, we carried out experiments using 4 types of input data which were captured from different factory environments and different machines. The training data set consisting of 50 defect points and 50 nondefect points were selected from said data and fed into the error back-propagation algorithm for training the network. Figure 10 shows that the pattern of convergence of error graph during back-propagation was perfect and the convergence limit (0.01) was very much acceptable.

By comparing the performance of our method with the existing thresholding method, following results were confirmed.

The existing ratio of miss-detections $(20 \% \sim 30 \%)$ was able to decrease around $5 \%$. This is because the candidate points to the network are selected by a low level threshold and the system has been trained to classify the waveform points by using only local features

The existing ratio of false detections $(20 \% \sim 30 \%)$ was able to decrease below $15 \%$. This is also due to the usage of a trained neural network for the purpose.

The manual workload for operators for threshold setting after any change of the machine itself or the environment or the location of the machine were able to slash. 
This proves that our method is more feasible and superior than the existing thresholding method for non-contact defect detection. Furthermore by selecting a training data set using every possible scenario, the miss-detection ratio and false detection ratio can be expected near zero

As the future direction of this research, we expect to continue this work for online training, which will enable any new entry to the network to be treated as a training data as well as input data simultaneously. Then the network will be able to perform the expected task while keeping the learning parameters updated whenever a new pattern of a defect point appears.

Adding another output to the neural network as "Uncertain" apart from the existing "OK" and "NG" is also one of our ideas in consideration for the next step. With recent demand of high density pitch patterning of TFT lines on flat panels the "Uncertain" judgment, which are required to inspect and decide visually by the operators, would become more common in automatic inspection systems in the industry.

\section{Acknowledgements}

The authors would like to express their gratitude to OHT Incorporation for their generous support and providing their actual data and all the resources and facilities for this research.

\section{References}

[1] Hiroshi Hamori, Masatoshi Sakawa, Hideki Katagiri, Takeshi Matsui. A Fast Non-Contact Inspection System based on Dual Channel Measurement System. Japan Institute of Electronics Packaging. 2010; 13(7): 562-568.

[2] Hiroshi Hamori, Masatoshi Sakawa, Hideki Katagiri, Takeshi Matsui. Fast Non-Contact flat Panel Inspection through a Duel Channel measurement System, Proceedings of International Conference on Computers and Industrial Engineering, CD-ROM, July 2010.

[3] Hiroshi Hamori, Masatoshi Sakawa, Hideki Katagiri, Takeshi Matsui, A Defect position Identification System based on a dual channel measurement system, Journal of Japan Institute of Electronics, Information and Communication Engineers. 2011; J94C(10): 323-333.

[4] Hiroshi Hamori, Masatoshi Sakawa, Hideki Katagiri, Takeshi Matsui, A Dual Channel Defect Position Identification Method for Touch Panel Manufacturing Process, Proceedings of International Conference on Electronics Packaging. 2011: $732-736$.

[5] Chi Jie Lu, Du Ming Tsai, Automatic Defect Inspection for LCD using Singular Value Decomposition, International journal of advanced manufacturing technology. 2005: 53-61. http://dx.doi.org/10.1007/s00170-003-1832-6

[6] Y.H. Liu, S.H. Lin, Y.L, Hsueh, M.J. Lee, Automatic target defect identification for TFT-LCD array process using kernel FCM based fuzzy SVDD ensemble, International journal of expert systems and applications. 2009; 36: 1978-1998, March 2009.

[7] Raul Rojas, Neural Networks - A Systematic Introduction, Springer-Verlag, 1996.

[8] D.E. Rumelhart, J.L. Mcclelland, Parallel distributed processing: Explorations in the microstructure of cognition. Volume 1. Foundations, The MIT Press.

[9] Guoqiang Peter Zhang, Neural Networks for Classification: A Survey, IEEE Transactions on System, Man and Cybernetics. 2000; 30(4): 451-462. http://dx.doi.org/10.1109/5326.897072 